\title{
Primary admission and secondary transfer of trauma patients to Dutch level I and level II trauma centers: predictors and outcomes
}

\author{
Claire R. L. van den Driessche ${ }^{1}$. Charlie A. Sewalt ${ }^{1,2} \oplus$. Jan C. van Ditshuizen ${ }^{1} \cdot$ Lisa Stocker $^{1,3}$.

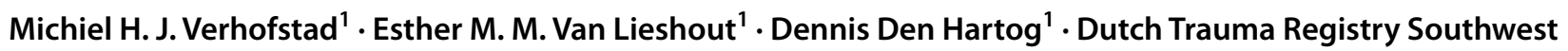

Received: 14 February 2021 / Accepted: 17 September 2021 / Published online: 29 September 2021

(c) The Author(s) 2021

\begin{abstract}
Purpose The importance and impact of determining which trauma patients need to be transferred between hospitals, especially considering prehospital triage systems, is evident. The objective of this study was to investigate the association between mortality and primary admission and secondary transfer of patients to level I and II trauma centers, and to identify predictors of primary and secondary admission to a designated level I trauma center.

Methods Data from the Dutch Trauma Registry South West (DTR SW) was obtained. Patients $\geq 18$ years who were admitted to a level I or level II trauma center were included. Patients with isolated burn injuries were excluded. In-hospital mortality was compared between patients that were primarily admitted to a level I trauma center, patients that were transferred to a level I trauma center, and patients that were primarily admitted to level II trauma centers. Logistic regression models were used to adjust for potential confounders. A subgroup analysis was done including major trauma (MT) patients (ISS > 15). Predictors determining whether patients were primarily admitted to level I or level II trauma centers or transferred to a level I trauma center were identified using logistic regression models.

Results A total of 17,035 patients were included. Patients admitted primarily to a level I center, did not differ significantly in mortality from patients admitted primarily to level II trauma centers (Odds Ratio (OR): 0.73; 95\% confidence interval (CI) 0.51-1.06) and patients transferred to level I centers (OR: 0.99; 95\%CI 0.57-1.71). Subgroup analyses confirmed these findings for MT patients. Adjusted logistic regression analyses showed that age (OR: 0.96; 95\%CI 0.94-0.97), GCS (OR: 0.81; 95\%CI 0.77-0.86), AIS head (OR: 2.30; 95\%CI 2.07-2.55), AIS neck (OR: 1.74; 95\%CI 1.27-2.45) and AIS spine (OR: 3.22; 95\% CI 2.87-3.61) are associated with increased odds of transfers to a level I trauma center.

Conclusions This retrospective study showed no differences in in-hospital mortality between general trauma patients admitted primarily and secondarily to level I trauma centers. The most prominent predictors regarding transfer of trauma patients were age and neurotrauma. These findings could have practical implications regarding the triage protocols currently used.
\end{abstract}

Keywords Transfer $\cdot$ Triage $\cdot$ Major trauma $\cdot$ Level $\cdot$ Trauma center $\cdot$ Predictors $\cdot$ Outcome

\section{Introduction}

Charlie A. Sewalt

c.sewalt@erasmusmc.nl

1 Trauma Research Unit, Department of Surgery, Erasmus MC University Medical Center Rotterdam, Doctor Molewaterplein 40, 3015 GD Rotterdam, The Netherlands

2 Center for Medical Decision Making, Department of Public Health, Erasmus MC University Medical Center Rotterdam, Doctor Molewaterplein 40, 3015 GD Rotterdam, The Netherlands

3 Department of Anesthesiology, Leiden University Medical Center, Albinusdreef 2, 2333 ZA Leiden, The Netherlands
Injuries are an important cause of morbidity and mortality, both in the developed world and the developing world [1]. Although the global burden of injuries has declined over the past years morbidity and mortality caused by injuries are still substantial [1]. To treat trauma patients, many countries have adopted a regionalized trauma network. Trauma care within the Netherlands is set up to contain designated trauma centers (TCs) spanning eleven trauma regions. Each region has a designated level I TC for the treatment of major trauma (MT) patients (Injury Severity Score (ISS) > 15) and level II and III centers for the stable patients. With such an 
exclusive trauma system survival rates of trauma patients have increased the past 15 years [2-5]. Within this system a trauma classification scheme (level I to III) has been established, with optimal 24/7 resources in level I TCs.

In the Netherlands, level I TCs are frequently academic teaching hospitals which have to meet minimum volume standards regarding the number of MT patients. Level II TCs offer less specialized trauma care and are not equipped for acute neuro-surgical procedures for head trauma and less prepared for severely injured patients $[6,7]$. This description highlights the differences between level I and level II TCs, however, currently there is no consensus concerning whether there is a difference in outcomes between level I and level II TCs within an established and mature trauma system [8-11].

Prehospital triage guidelines are important to ensure trauma patients are admitted as quick as possible to their respective TCs. Although this concept seems simple, the decision to which hospital, and thus the right level of care a patient needs to be transported is made by emergency medical service (EMS) providers. Compliance rates of triage protocols and experience of EMS providers are decisive to make the right judgement and dependent of a large number of variables, such as assessment of injury severity and local health care context.

Whether injured trauma patients have worse outcomes when secondarily admitted via transfer to a level I TC instead of being primarily admitted is unclear [12, 13]. Some studies suggest a difference, reporting patients with traumatic brain injury and severe injuries $[14,15]$ have a survival benefit when primarily admitted to a level I TC. In contrast, other studies found no difference in outcomes between level I and level II TCs (8), examined transfers without comparison to primary admission [16] or analyzed level I and level II TCs combined $[15,16]$.

The aim of this study is to compare in-hospital mortality between trauma patients primarily admitted to a level I TC, trauma patients primarily admitted to a level II TC and patients secondarily transferred to a level I TC. The secondary aim was to identify predictors for primary admission to a level I or level II TC, and for secondary admission from level II to level I TCs via transfer.

\section{Methods}

\section{Study design}

Data for this study were obtained from trauma region Southwest of the Dutch National Trauma Registry (DNTR), which is a database that is maintained by 11 administrative TCs nationwide. The DNTR handle general inclusion criteria; all patients admitted to the emergency department (ED) within $48 \mathrm{~h}$ after trauma, followed by either hospitalization, transfer to other hospitals or death are included, excluding patients that are dead on arrival. Information on patient demographics, prehospital care and injuries are coded using the Abbreviated Injury Scale (AIS), and outcomes are registered [17]. In total, the region Southwest Netherlands consists of one Level I TC and five Level II TCs.

A retrospective cohort study was performed including all trauma patients $\geq 18$ years who were admitted to the level I or level II TCs between January 12015 and December 31 2018. Transfer is defined as primarily presented at a level II TC and transferred to a level I TC within $48 \mathrm{~h}$. Patients with isolated burn injuries were excluded because these patients are treated at one of the three nationally coordinated burn centers, of which one is located within the trauma region Southwest. Patients treated in level III TCs or originating from level III TCs were excluded due to the large difference in case mix of patients presented and/or admitted to level III TCs compared to patients admitted to level I and II TCs.

The primary outcome measure was in-hospital mortality.

\section{Type of Hospital Admission: primary and secondary}

Patients were divided into three groups: trauma patients presented and admitted at the level I TC (PA level I), trauma patients primarily presented at a level II TC followed by a secondary transfer to the level I TC (ST level I), and trauma patients presented and admitted at a level II TC (PA level II).

\section{Statistical analysis}

First, a descriptive analysis was executed for the three patient groups regarding patient characteristics, injury characteristics and outcome characteristics. For continuous and ordinal variables, medians with 25 th and 75 th percentiles were reported. For nominal variables, frequencies with percentages were reported. The transfer group was further divided into ISS $>15$ and ISS $<15$ for a subgroup analysis.

Second, missing values were imputed with multilevel multiple imputation dependent on mechanism of missingness [18-20]. Outcome measures were not imputed since these variables had no missing values.

Third, a random effects logistic regression model was made to evaluate the association between type of hospital admission and in-hospital mortality. This provided an unadjusted estimate. After that, we added the following confounders to the model: age, gender, mechanism of injury, ISS, and prehospital vital parameters (systolic blood pressure (SBP), Glasgow Coma Scale (GCS), respiratory rate (RR)). Additionally, an ISS > 15 (MT) subgroup analysis was done. Continuous variables were tested for non-linearity using restricted cubic splines. When non-linearity was assumed, variables were split into two continuous 
linear variables with the optimal cut-off achieved from the restricted cubic spline [21].

To identify predictors determining whether trauma patients are primarily admitted to a level I or level II TC and to identify what predictors determine secondary admission to the level I TC univariate analyses were performed using two logistic regression models, one for the outcome primary admission to level I versus secondary transfer to level I and one for the outcome primary admission to level II versus secondary transfer from level II to level I. The following variables were analyzed for an association with hospital admission: age (continuous), sex, mechanism of injury, AIS scores for head, chest, abdomen and extremities (continuous), and prehospital vital parameters (SBP, GCS, RR, continuous).

After that, two multivariable logistic regression models were created, one with outcome primary admission to level I versus secondary transfer to level I and one for the outcome primary admission to level II versus secondary transfer from level II tot level I, in which the following variables were analyzed for an association with hospital admission: age (continuous), sex, mechanism of injury, AIS scores for head, chest, abdomen and extremities (continuous), and prehospital vital parameters (SBP, GCS, RR, continuous). Continuous predictors were tested for non-linearity using restricted cubic splines and non-linear variables were cut into categories based on the restricted cubic spline.

Data were analyzed using the R Software Environment (version 3.5.1, the R Foundation for Statistical Computing, Vienna Austria). This study was done in accordance with the STROBE Statement.

\section{Results}

\section{Study characteristics}

A total of 17,035 records of trauma patients were included, of which 3658 patients were primarily admitted to a level I TC, 301 patients were transferred from a level II to a level I TC, and 13,076 patients were primarily admitted to a level II TC (Fig. 1). All patients were transferred within $48 \mathrm{~h}$ and $276(92 \%)$ were transferred within $6 \mathrm{~h}$ to a Level I TC.

Patients primarily admitted to level II TCs tended to be older than patients transferred or primarily admitted to a level I TC (Table 1$)$. Males $(n=2589,70.8 \%)$ were more often primarily admitted to a level I TC or transferred to a level I TC than females $(n=1069,29.2 \%)$. Patients with a GCS of $\leq 8$ seem to mostly be either primarily admitted to a level I TC or transferred to a level I TC. Patients with an ISS $>15$ (1768) tend to be primarily admitted to a level I TC or transferred to a level I TC; a total of 1206 (68.2\%) patients with an ISS $>15$ are primarily admitted to a level I TC and a total of $1358(76.8 \%)$ patients when transfers are added.
Patients primarily admitted to a level I TC or transferred to a level I TC had a higher unadjusted mortality rate. Patients transferred to level I TCs with an ISS $<15$ had less signs of shock $(\mathrm{SBP}<90)$ and were less severely injured.

\section{Impact on outcome}

The overall mortality was 3.9\% (Table 1). The highest unadjusted mortality occurred in primary admissions to level I TCs $(8.4 \%)$, followed by the secondary transfer group (7.6\%) and primary admissions to level II TCs (2.6\%). Univariate analysis shows a lower mortality for primary admissions to level II TCs compared to primary admissions to level I TCs (OR, 0.29; 95\% CI, 0.23-0.37; $p<0.001$, Table 2). Multivariable analysis adjusting for gender, age, GCS, RR, SBP, ISS, and mechanism of injury showed that the adjusted mortality in the transfer group and primary admission to level II TCs group did not differ significantly from the primary admission to level I TCs group. When solely focusing on MT patients, univariate analysis showed a lower mortality for both transfer patients as well as primary admissions to level II TCs. Multivariable analysis for MT patients showed that the adjusted mortality in the transfer group (adjusted OR, $0.72 ; 95 \% \mathrm{CI}, 0.40-1.31 ; p=0.28)$ and primary admission to level II TCs group (adjusted OR, $0.70 ; 0.45-1.11 ; p=0.13$ ) did not differ significantly from the primary admission to the level I TC group (Table 2).

\section{Predictors of transfer}

Univariate analyses comparing patients transferred to the level I TC to patients primarily admitted to level I TCs showed that transfer patients are more likely to be older and are less likely to be male (Table 3 ). When conducting multivariable analyses comparing patients transferred to level I TCs to patients primarily admitted to level I TCs, transfer patients were found more likely to have head injuries (adjusted OR, 1.37; 95\% CI, 1.25-1.50) and spine injuries (adjusted OR, 1.61; 95\% CI, 1.47-1.76). Additionally, transfer patients are more likely to have face injuries and (superficial) skin injuries (Table 3).

When comparing patients transferred to the level I TCs to patients admitted primarily to level II TCs univariate analyses showed that, transfer patients are more likely to be male and are less likely to have lower extremities injuries. Multivariable analyses adjusting for other possible predictors showed that patients transferred to level I TCs are less likely to be older $(\geq 70)$, are more likely to have a GCS $<8$ (adjusted OR, 0.81; 95\% CI, 0.76-0.85) and are found more likely to have head injuries (adjusted OR, 2.36; 95\% CI, 2.04-2.50) compared to patients admitted primarily to level II TCs (Table 4). 
Fig. 1 Flowchart

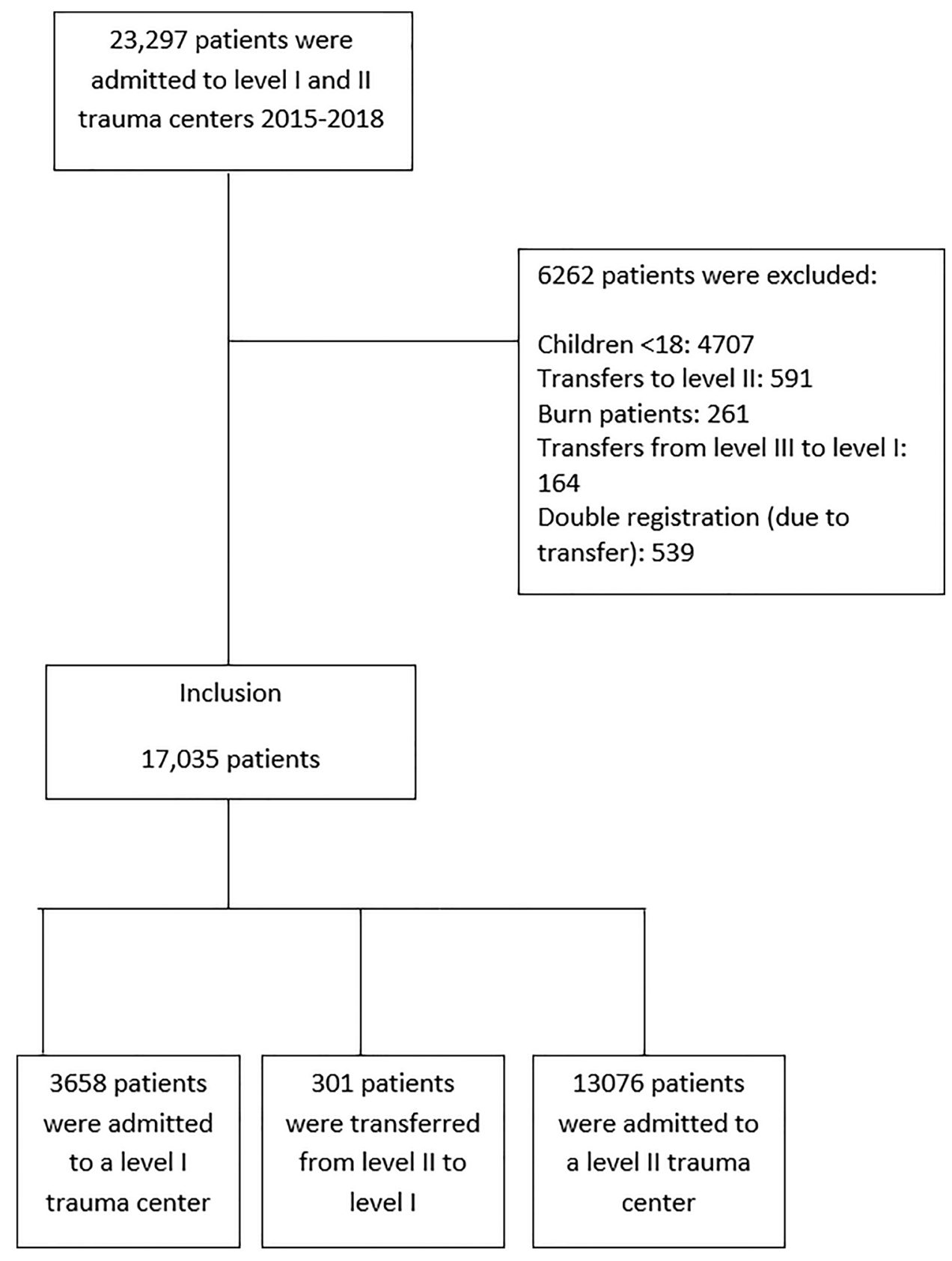

\section{Discussion}

The present study demonstrated no difference for in-hospital mortality between patients that are primarily admitted at level I TCs, patients that are transferred from level II to level I TCs, and patients that are primarily admitted at level II TCs. Furthermore, the present study found several possible predictors regarding type of admission of trauma patients. Compared to patients primarily presented at level I TCs, transferred patients were found more likely to have a higher GCS, a lower SBP, more severe head and spine injuries, and less severe face, thorax, lower extremities and skin injuries. In addition, transferred patients compared to patients primarily presented at level II TCs, were more likely to be younger, have a lower GCS, more severe head, neck, spine and abdominal injuries.

Differences in clinical outcomes measures between level I and level II TCs have been studied in the past [22, 23]. The present study demonstrated similar in-hospital mortality for level I and level II TCs, and transfer patients. These findings were previously reported for general trauma populations [22, 23]. Other studies did find a difference in mortality between level I and level II TCs [6, 11, 24]. A possible explanation for such contradicting results is that trauma systems in high-income countries have matured. Additionally, it is possible that geographical factors play a more prominent 
Table 1 Baseline characteristics for patients primarily admitted to level I TCs $(N=3658)$, transferred to level I TCs $(N=301)$ and primarily admitted to level II TCs $(N=13,076)$

\begin{tabular}{|c|c|c|c|c|}
\hline & Level I $(N=3658)$ & $\begin{array}{l}\text { Transfer to level } \\
\text { I }(N=301)\end{array}$ & Level II $(N=13,076)$ & $p$-value \\
\hline \multicolumn{5}{|l|}{ Patient characteristics } \\
\hline Male $(\%)$ & $2589(70.8)$ & $193(64.1)$ & $5656(43.3)$ & $<0.001$ \\
\hline Age (median [p25-p75]) & $49[31-66]$ & $58[40-70]$ & $75[57-85]$ & $<0.001$ \\
\hline Mechanism of injury (\%) & & & & $<0.001$ \\
\hline Traffic: motorized vehicle & $329(9.0)$ & $20(6.6)$ & $468(3.6)$ & \\
\hline Traffic: motorcycle & $132(3.6)$ & $5(1.7)$ & $141(1.1)$ & \\
\hline Traffic: moppet/scooter & $238(6.5)$ & $19(6.3)$ & $408(3.1)$ & \\
\hline Traffic: bike & $462(12.6)$ & $36(12.0)$ & $1425(10.9)$ & \\
\hline Traffic: pedestrian & $164(4.5)$ & $9(3.0)$ & $162(1.2)$ & \\
\hline Traffic: other & $31(0.8)$ & $2(0.7)$ & $72(0.6)$ & \\
\hline Shooting incident & $89(2.4)$ & $2(0.7)$ & $20(0.2)$ & \\
\hline Stabbing & $182(5.0)$ & $5(1.7)$ & $114(0.9)$ & \\
\hline Blunt object trauma & $200(5.5)$ & $15(5.0)$ & $260(2.0)$ & \\
\hline Fall on same level & $1063(29.1)$ & $132(43.9)$ & $9151(70.0)$ & \\
\hline Fall from higher level & $341(9.3)$ & $26(8.6)$ & $327(2.5)$ & \\
\hline Explosion & $18(0.5)$ & $0(0.0)$ & $12(0.1)$ & \\
\hline Drowning & $37(1.0)$ & $0(0.0)$ & $13(0.1)$ & \\
\hline Asphyxiation & $28(0.8)$ & $1(0.3)$ & $34(0.3)$ & \\
\hline Other & $344(9.4)$ & $29(9.6)$ & $469(3.6)$ & \\
\hline $\begin{array}{l}\text { Systolic blood pressure (median } \\
\text { [p25-p75]) }\end{array}$ & 135 [116-154] & 140 [121-155] & $141[121-161]$ & $<0.001$ \\
\hline $\mathrm{SBP}<90(\%)$ & $188(5.9)$ & $3(1.2)$ & $154(1.3)$ & $<0.001$ \\
\hline GCS (median [p25-p75]) & 15 [13-15] & $15[14,15]$ & $15[15-15]$ & $<0.001$ \\
\hline $\mathrm{GCS} \leq 8(\%)$ & $554(15.7)$ & $36(13.5)$ & $81(0.8)$ & $<0.001$ \\
\hline RR (median [p25-p75]) & $15[12-20]$ & $15[12-18]$ & $15[12-18]$ & 0.001 \\
\hline \multicolumn{5}{|l|}{ Injury characteristics } \\
\hline ISS (median [p25-p75]) & $9[5-18]$ & $16[9-22]$ & 9 [4-9] & $<0.001$ \\
\hline ISS > $15(\%)$ & $1206(33.0)$ & $152(50.5)$ & $410(3.1)$ & $<0.001$ \\
\hline AIS head $\geq 3(\%)$ & $934(25.5)$ & $125(41.5)$ & $624(4.8)$ & $<0.001$ \\
\hline AIS thorax $\geq 3(\%)$ & $660(18.0)$ & $36(12.0)$ & $537(4.1)$ & $<0.001$ \\
\hline AIS abdomen $\geq 3(\%)$ & $143(3.9)$ & $9(3.0)$ & $74(0.6)$ & $<0.001$ \\
\hline AIS upper extremities $\geq 3(\%)$ & $77(2.1)$ & $4(1.3)$ & $53(0.4)$ & $<0.001$ \\
\hline AIS lower extremities $\geq 3(\%)$ & $587(16.0)$ & $22(7.3)$ & $5404(41.3)$ & $<0.001$ \\
\hline \multicolumn{5}{|l|}{ Outcome characteristics } \\
\hline In-hospital mortality (\%) & $309(8.4)$ & $23(7.6)$ & $342(2.6)$ & $<0.001$ \\
\hline LOS ICU (median [p25-p75]) & $0[0-0]$ & $0[0-2]$ & $0[0-0]$ & $<0.001$ \\
\hline LOS (median [p25-p75]) & $4.0[2.0-10.0]$ & $5.5[3.0-11.0]$ & $5.0[2.0-9.0]$ & $<0.001$ \\
\hline
\end{tabular}

Categorical variables represented with $n(\%)$; continuous variables represented with median [p25-p75]; p25-p75, 25th percentile-75th percentile; GCS Glasgow coma score, ISS injury severity score, AIS abbreviated injury scale, $I C U$ intensive care unit, $L O S$ length of stay role in larger countries in patient allocation. Another possible explanation is that analyses were limited by case-mix differences, therefore not adjusting sufficiently for confounders. The present study found no difference for in-hospital mortality in MT patients. This is not in accordance with findings by other studies [23]. A possible explanation is that while the trauma system in the Netherlands has been maturing, and along with public health in general, mortality rates have steadily been decreasing, also among MT patients.
Also Dutch Level II TCs do not have neurosurgery available which explains the high amount of secondary referred neurotrauma patients. Studies often exclude transfer patients, the studies that do include transfer patients are in agreement with the findings of the current study and find no difference in outcomes in patients primarily and secondarily admitted to level I and level II TCs [12, 13, 25, 26].

This study found several possible predictors regarding admission of trauma patients. Compared to patients 
Table 2 Odds ratios in-hospital mortality of transferred patients $(N=301)$ and patients primarily admitted to level II TCs $(N=13,076)$ compared to patients primarily admitted to level I TCs $(N=3658)$
Table 3 Predictors for being transferred: odds ratios of transfer patients $(N=301)$ compared to patients primarily admitted to level I TCs $(N=3658)$

\begin{tabular}{lcccc}
\hline & Unadjusted OR $(95 \% \mathrm{CI})$ & P-value & Adjusted OR (95\% CI) & $P$-value \\
\hline Transfer & $0.90(0.58-1.39)$ & 0.63 & $0.99(0.57-1.71)$ & 0.97 \\
Level 2 & $0.29(0.23-0.37)$ & $<0.001$ & $0.73(0.51-1.06)$ & 0.10 \\
$I S S>15$ & & & & \\
Transfer & $0.50(0.30-0.81)$ & 0.01 & $0.72(0.40-1.31)$ & 0.28 \\
Level 2 & $0.49(0.35-0.67)$ & $<0.001$ & $0.70(0.45-1.11)$ & 0.13 \\
\hline
\end{tabular}

*Adjusted for age, gender, mechanism of injury, ISS, prehospital systolic blood pressure, prehospital GCS and prehospital respiratory rate

OR odds ratio, 95\% CI 95\% confidence interval, GCS Glasgow coma scale

\begin{tabular}{lcc}
\hline & Univariable OR (95\% CI) & Multivariable* OR (95\% CI) \\
\hline Gender (male) & $0.74(0.58-0.94)$ & $0.80(0.61-1.05)$ \\
Age & $1.01(1.01-1.02)$ & $1.01(0.999-1.014)$ \\
GCS & $1.02(0.99-1.06)$ & $1.05(1.00-1.10)$ \\
Respiratory rate $<18$ & $1.07(0.99-1.15)$ & $1.06(0.99-1.15)$ \\
Respiratory rate $\geq 18$ & $0.87(0.78-0.97)$ & $0.91(0.82-1.01)$ \\
Systolic blood pressure $<140$ & $1.01(1.00-1.02)$ & $1.01(1.00-1.03)$ \\
Systolic blood pressure $\geq 140$ & $0.99(0.981-0.999)$ & $0.99(0.976-0.997)$ \\
AIS head & $1.19(1.11-1.26)$ & $1.37(1.25-1.50)$ \\
AIS face & $0.74(0.62-0.88)$ & $0.63(0.52-0.76)$ \\
AIS neck & $1.00(0.77-1.30)$ & $1.08(0.81-1.44)$ \\
AIS spine & $1.43(1.33-1.55)$ & $1.61(1.47-1.76)$ \\
AIS thorax & $0.81(0.72-0.90)$ & $0.76(0.67-0.87)$ \\
AIS abdomen & $0.87(0.73-1.04)$ & $1.11(0.91-1.34)$ \\
AIS upper extremities & $0.84(0.72-0.97)$ & $0.97(0.83-1.13)$ \\
AIS lower extremities & $0.76(0.67-0.85)$ & $0.85(0.75-0.96)$ \\
AIS external & $0.57(0.40-0.82)$ & $0.60(0.40-0.91)$ \\
Mechanism of injury & $0.40(0.15-1.10)$ & $0.44(0.15-1.31)$ \\
Traffic: pedestrian & $0.91(0.63-1.30)$ & $0.65(0.39-1.06)$ \\
Traffic: bike & $0.71(0.40-1.26)$ & $0.62(0.36-1.06)$ \\
Traffic: motorized vehicle $($ car, motor- & & $1.00(0.56-1.81)$ \\
cycle) & $0.95(0.59-1.53)$ & $0.62(0.37-1.04)$ \\
Traffic: scooter/mopet & $0.86(0.58-1.28)$ & $1.61(0.91-2.85)$ \\
High energy fall & $1.68(1.32-2.14)$ & \\
Low energy fall & $0.81(0.59-1.13)$ & \\
Other & & \\
\hline & &
\end{tabular}

*Adjusted for all other predictors in this analysis

OR odds ratio, 95\% CI, 95\% confidence interval, GCS Glasgow coma scale, AIS abbreviated injury scale Cut-off values of non-linear variables were based on restricted cubic splines primarily presented at level I TCs, patients transferred from a level II TC to a level I TC are more likely to have a higher GCS, a lower SBP, more severe head and spine injuries, and less severe facial, thorax, lower extremities and skin injuries. If compliance with prehospital triage is assumed, this demonstrates that patients with unnoticed or not yet apparent signs of TBI are more likely to be transferred when first admitted to a level II TC. This assumption is consistent with previous studies describing that most deaths caused by undertriage are secondary to severe TBI [27].

Compared to patients primarily presented and admitted to level II TCs, transferred patients were more likely to be younger, have a lower GCS and more severe head, neck, spine and abdominal injuries, indicating injuries of the central nervous system. Other studies found similar results, showing that transferred patients presented with more severe head and abdominal injuries and with lower GCS $[16,26]$. 
Table 4 Predictors for being transferred: odds ratios of transfer patients $(N=301)$ compared to patients primarily admitted to level II TCs $(N=13,076)$

\begin{tabular}{lcl}
\hline & $\begin{array}{l}\text { Univariable } \\
\text { OR }(95 \% \text { CI })\end{array}$ & $\begin{array}{l}\text { Multivariable* } \\
\text { OR }(95 \% \text { CI })\end{array}$ \\
\hline Gender (male) & $2.34(1.85-2.98)$ & $1.11(0.83-1.48)$ \\
Age $<70$ & $1.00(0.99-1.01)$ & $1.00(0.99-1.01)$ \\
Age $\geq 70$ & $0.95(0.94-0.96)$ & $0.96(0.94-0.97)$ \\
GCS & $0.74(0.71-0.77)$ & $0.81(0.76-0.85)$ \\
RR & $0.99(0.96-1.02)$ & $0.99(0.96-1.02)$ \\
SBP $<140$ & $1.01(0.996-1.018)$ & $1.01(1.00-1.03)$ \\
SBP $\geq 140$ & $0.99(0.977-0.999)$ & $0.99(0.98-1.00)$ \\
AIS head & $2.21(2.06-2.37)$ & $2.36(2.04-2.50)$ \\
AIS face & $1.73(1.45-2.05)$ & $0.85(0.68-1.06)$ \\
AIS neck & $2.26(1.69-3.01)$ & $1.77(1.26-2.48)$ \\
AIS spine & $2.77(2.54-3.03)$ & $3.20(2.86-3.59)$ \\
AIS thorax & $1.37(1.22-1.53)$ & $1.00(0.86-1.17)$ \\
AIS abdomen & $1.57(1.33-1.87)$ & $1.43(1.16-1.77)$ \\
AIS upper extremities & $1.15(0.997-1.329)$ & $1.06(0.88-1.26)$ \\
AIS lower extremities & $0.48(0.43-0.54)$ & $1.01(0.87-1.16)$ \\
AIS external & $1.16(0.87-1.56)$ & $0.70(0.47-1.06)$ \\
Mechanism of injury & & \\
Traffic: pedestrian & $1.93(0.70-5.34)$ & $1.30(0.43-3.93)$ \\
Traffic: bike & $1.14(0.80-1.64)$ & $0.35(0.20-0.61)$ \\
Traffic: motorized vehicle & $0.54(0.31-0.95)$ & $0.52(0.30-0.93)$ \\
Traffic: scooter/mopet & $2.14(1.33-3.44)$ & $0.51(0.27-0.99)$ \\
High energy fall & $3.63(2.46-5.38)$ & $0.46(0.25-0.83)$ \\
Low energy fall & $0.34(0.27-0.44)$ & $0.36(0.23-0.57)$ \\
Other & $2.68(1.94-3.72)$ & $2.63(1.41-4.93)$ \\
\hline & &
\end{tabular}

*Adjusted for all other predictors in this analysis

OR odds ratio, $95 \%$ CI, 95\% confidence interval, GCS Glasgow coma scale, AIS abbreviated injury scale

Cut-off values of non-linear variables were based on restricted cubic splines

The findings concerning age could be explained by a study which found nearly half of older trauma patients to be undertriaged [28]. However, another study found transferred patients to be older [26]. These different findings might be due to undertriage of older trauma patients resulting in these patients staying in non-level I TCs whereas discovery of these undertriaged patients could result in an increase of transfers.

Prehospital identification of MT patients and identification of MT patients at the ED could better focus on specific subgroups that are often undertriaged. The results show that triage leading to admission to a level 1 TC does not always coincide with an ISS $>15$. This raises the question whether the ISS $>15$ is a reliable tool regarding the identification of patients that are in need of an admission to level I TCs. A possible alternative suggestion could be a more multidimensional approach by not just taking into account the three body regions with the highest AIS scores as done when calculating the ISS but to also asses which body regions have AIS scores above zero, paying particular attention to head, neck and spine. An example of this is the New Injury Severity Scale (NISS), which takes into account the three highest AIS scores regardless of body region whereas the ISS takes into account the three highest AIS scores from the three different most severely injured body regions. The NISS has been proven to identify more MT patients than the ISS [29]. The trouble with identifying patients that need level I trauma care and patients that need level II trauma care or lower level care is a phenomenon that encompasses many countries and triage protocols, as findings show nearly all triage protocols are unable to properly identify severely injured patients [30].

\section{Limitations}

The present study included validated data of a large number of patient records. However, the retrospective design is traditionally limited with several biases. The biggest limitation of our study is the fact that there could be a negative selection bias because transferred patients tend to have worser outcomes than level II TC patients since that is the reason for a transfer to a Level I TC. In non-randomized trials, the observed study results observed may be because of unmeasured factors or variables. There is a chance of confounding bias due to case-mix differences. Additionally, the sample size of the transferred patient group could be too small and therefore lacking in power. Results of the transfer group should be interpreted with caution, especially regarding in-hospital mortality. It is possible there is a statistically significant difference in mortality that was not detected due to the small sample size. Another limitation is focusing on in-hospital mortality only Further research regarding outcomes such as quality of life and functional outcomes could also provide more information about which level of trauma care is appropriate for certain trauma populations.

\section{Conclusions}

The current study has provided several possible predictors regarding transfer of trauma patients, most prominent are age and neurotrauma. These findings could have practical implications regarding the triage protocol currently used. Half of the patients transferred to the level I TC have an ISS $<15$. It is possible a move away from the ISS $>15$ and the weight this holds to other parameters could improve triage and therefore, admittance of trauma patients to the right level of center. Focus should better be on specific subgroups that are currently under triaged and primarily admitted to other levels of trauma care. 
Acknowledgements Author collaborator group Dutch Trauma Registry Southwest: J.M. van Buijtenen: Department of Surgery, Sint Franciscus Gasthuis, Kleiweg 500, 3045 PM, Rotterdam, The Netherlands. P.T. den Hoed: Department of Surgery, Ikazia Ziekenhuis, Montessoriweg 1, 3083 AN, Rotterdam, The Netherlands. T.S.C. Jakma: Department of Surgery, Albert Schweitzer Ziekenhuis, Albert Schweitzerplaats 25, 3318 AT, Dordrecht, The Netherlands. G. de Klerk: Department of Surgery, Admiraal de Ruijter Ziekenhuis, 's-Gravenpolderseweg 114, 4462 RA, Goes, The Netherlands. G.R. Roukema: Department of Surgery, Maasstad Ziekenhuis, Maasstadweg 21, 3079 DZ, Rotterdam, The Netherlands.

Author contributions All authors contributed to the study conception and design. Data collection and analysis were performed by Claire van den Driessche, Charlie Sewalt and Jan van Ditshuizen. The first draft of the manuscript was written by Claire van den Driessche and Charlie Sewalt and all authors commented on previous versions of the manuscript. All authors read and approved the final manuscript.

Funding The authors did not receive support from any organization for the submitted work.

Availability of data and materials Data can be made available upon request.

Code availability $\mathrm{R}$ code can be made available upon request.

\section{Declarations}

Conflict of interest The authors have no relevant financial or non-financial interests to disclose.

Ethics approval Ethical approval was waived by the local Ethics Committee of Erasmus Medical Center in view of the retrospective nature of the study and all the procedures being performed were part of the routine care.

Consent to participate Not applicable due to the retrospective nature of this study.

Consent for publication Not applicable due to the retrospective nature of this study.

Open Access This article is licensed under a Creative Commons Attribution 4.0 International License, which permits use, sharing, adaptation, distribution and reproduction in any medium or format, as long as you give appropriate credit to the original author(s) and the source, provide a link to the Creative Commons licence, and indicate if changes were made. The images or other third party material in this article are included in the article's Creative Commons licence, unless indicated otherwise in a credit line to the material. If material is not included in the article's Creative Commons licence and your intended use is not permitted by statutory regulation or exceeds the permitted use, you will need to obtain permission directly from the copyright holder. To view a copy of this licence, visit http://creativecommons.org/licenses/by/4.0/.

\section{References}

1. Haagsma JA, Graetz N, Bolliger I, Naghavi M, Higashi H, Mullany EC, et al. The global burden of injury: incidence, mortality, disability-adjusted life years and time trends from the Global Burden of Disease study 2013. Inj Prev. 2016;22(1):3-18.

2. Ashley DW, Pracht EE, Medeiros RS, Atkins EV, NeSmith EG, Johns TJ, et al. An analysis of the effectiveness of a state trauma system: treatment at designated trauma centers is associated with an increased probability of survival. J Trauma Acute Care Surg. 2015;78(4):706-12 (discussion 12-4).

3. Mullins RJ, Veum-Stone J, Hedges JR, Zimmer-Gembeck MJ, Mann NC, Southard PA, et al. Influence of a statewide trauma system on location of hospitalization and outcome of injured patients. J Trauma. 1996;40(4):536-45 (discussion 45-6).

4. Celso B, Tepas J, Langland-Orban B, Pracht E, Papa L, Lottenberg $\mathrm{L}$, et al. A systematic review and meta-analysis comparing outcome of severely injured patients treated in trauma centers following the establishment of trauma systems. J Trauma. 2006;60(2):371-8 (discussion 8).

5. MacKenzie EJ, Rivara FP, Jurkovich GJ, Nathens AB, Frey KP, Egleston BL, et al. A national evaluation of the effect of traumacenter care on mortality. N Engl J Med. 2006;354(4):366-78.

6. Demetriades D, Martin M, Salim A, Rhee P, Brown C, Chan $\mathrm{L}$. The effect of trauma center designation and trauma volume on outcome in specific severe injuries. Ann Surg. 2005;242(4):512-7 (discussion 7-9).

7. Demetriades D, Martin M, Salim A, Rhee P, Brown C, Doucet $\mathrm{J}$, et al. Relationship between American College of Surgeons trauma center designation and mortality in patients with severe trauma (injury severity score $>15$ ). J Am Coll Surg. 2006;202(2):212-5 (quiz A45).

8. Rogers FB, Osler T, Lee JC, Sakorafas L, Wu D, Evans T, et al. In a mature trauma system, there is no difference in outcome (survival) between Level I and Level II trauma centers. J Trauma. 2011;70(6):1354-7.

9. Cudnik MT, Newgard CD, Sayre MR, Steinberg SM. Level I versus Level II trauma centers: an outcomes-based assessment. J Trauma. 2009;66(5):1321-6. https://doi.org/10.1097/TA.0b013 e3181929e2b.

10. McConnell KJ, Newgard CD, Mullins RJ, Arthur M, Hedges JR. Mortality benefit of transfer to level I versus level II trauma centers for head-injured patients. Health Serv Res. 2005;40(2):435-57.

11. Kaji AH, Bosson N, Gausche-Hill M, Dawes AJ, Putnam B, Shepherd T, et al. Patient outcomes at urban and suburban level I versus level II trauma centers. Ann Emerg Med. 2017;70(2):161-8.

12. Hill AD, Fowler RA, Nathens AB. Impact of interhospital transfer on outcomes for trauma patients: a systematic review. J Trauma. 2011;71(6):1885-900. https://doi.org/10.1097/TA.0b013e3182 3 ac642.

13. Williams T, Finn J, Fatovich D, Jacobs I. Outcomes of different health care contexts for direct transport to a trauma center versus initial secondary center care: a systematic review and meta-analysis. Prehosp Emerg Care. 2013;17(4):442-57. https://doi.org/10. 3109/10903127.2013.804137.

14. Garwe T, Cowan LD, Neas BR, Sacra JC, Albrecht RM. Directness of transport of major trauma patients to a level I trauma center: a propensity-adjusted survival analysis of the impact on short-term mortality. J Trauma. 2011;70(5):1118-27. https://doi. org/10.1097/TA.0b013e3181e243b8.

15. Hartl R, Gerber LM, Iacono L, Ni Q, Lyons K, Ghajar J. Direct transport within an organized state trauma system reduces mortality in patients with severe traumatic brain injury. $\mathrm{J}$ Trauma. 2006;60(6):1250-6.

16. Adzemovic T, Murray T, Jenkins P, Ottosen J, Iyegha U, Raghavendran K, et al. Should they stay or should they go? Who benefits from interfacility transfer to a higher-level trauma center following initial presentation at a lower-level trauma center. J Trauma Acute Care Surg. 2019;86(6):952-60. 
17. LNAZ. Landelijk Netwerk Acute Zorg: Landelijke Trauma Registratie (LTR). 2020.

18. Donders AR, van der Heijden GJ, Stijnen T, Moons KG. Review: a gentle introduction to imputation of missing values. J Clin Epidemiol. 2006;59(10):1087-91. https://doi.org/10.1016/j.jclinepi. 2006.01.014.

19. Li P, Stuart EA, Allison DB. Multiple Imputation: A flexible tool for handling missing data. JAMA. 2015;314(18):1966-7. https:// doi.org/10.1001/jama.2015.15281.

20. Enders CK. Multiple imputation as a flexible tool for missing data handling in clinical research. Behav Res Ther. 2017;98:4-18. https://doi.org/10.1016/j.brat.2016.11.008.

21. Gauthier J, Wu QV, Gooley TA. Cubic splines to model relationships between continuous variables and outcomes: a guide for clinicians. Bone Marrow Transplant. 2020;55(4):675-80. https:// doi.org/10.1038/s41409-019-0679-x.

22. Kim YJ. Relationship of trauma centre characteristics and patient outcomes: a systematic review. J Clin Nurs. 2014;23(3-4):301-14. https://doi.org/10.1111/jocn.12129.

23. Van Ditshuizen JC, Van Den Driessche CRL, Sewalt CA, Van Lieshout EMM, Verhofstad MHJ, Den Hartog D. The association between level of trauma care and clinical outcome measures: a systematic review and meta-analysis. J Trauma Acute Care Surg. 2020 Oct;89(4):801-812.

24. Sethi D, Aljunid S, Saperi SB, Zwi AB, Hamid H, Mustafa AN, et al. Comparison of the effectiveness of major trauma services provided by tertiary and secondary hospitals in malaysia. $\mathrm{J}$ Trauma. 2002;53(3):508-16.

25. Wahle-Gerhardt A, Winkelmann M, Mommsen P, Krettek C, Zeckey C. Interhospital transfer of severely injured trauma patients does not influence outcome. Emergencias. 2018;30(4):253-60.

26. Hamada SR, Delhaye N, Degoul S, Gauss T, Raux M, Devaud ML, et al. Direct transport vs secondary transfer to level I trauma centers in a French exclusive trauma system: Impact on mortality and determinants of triage on road-traffic victims. PLoS ONE. 2019;14(11): e0223809. https://doi.org/10.1371/journal.pone. 0223809 .

27. Schellenberg M, Benjamin E, Bardes JM, Inaba K, Demetriades D. Undertriaged trauma patients: Who are we missing? J Trauma Acute Care Surg. 2019;87(4):865-9.

28. Uribe-Leitz T, Jarman MP, Sturgeon DJ, Harlow AF, Lipsitz SR, Cooper Z, Salim A, Newgard CD, Haider AH. National Study of Triage and Access to Trauma Centers for Older Adults. Ann Emerg Med. 2020;75(2):125-135.

29. Whitaker IY, Gennari TD, Whitaker AL. The difference between ISS and NISS in a series of trauma patients in Brazil. Annu Proc Assoc Adv Automot Med. 2003;47:301-9.

30. van Rein EAJ, Houwert RM, Gunning AC, Lichtveld RA, Leenen LPH, van Heijl M. Accuracy of prehospital triage protocols in selecting severely injured patients: a systematic review. J Trauma Acute Care Surg. 2017;83(2):328-39. 